\title{
Diagnostic spectroscopy of G-band brightenings in the photosphere of the sun
}

\author{
K. Langhans ${ }^{1,2}$, W. Schmidt ${ }^{1}$, and T. Rimmele ${ }^{3}$ \\ 1 Kiepenheuer-Institut für Sonnenphysik, Schöneckstr. 6, 79104 Freiburg, Germany \\ e-mail: wolfgang@kis.uni-freiburg.de \\ 2 The Institute for Solar Physics of the Royal Swedish Academy of Sciences, AlbaNova University Centre, \\ 10691 Stockholm, Sweden \\ e-mail: kai@astro.su.se \\ 3 National Solar Observatory, PO Box 62, Sunspot, NM 88349, USA \\ e-mail: rimmele@nso.edu
}

Received 16 December 2003 / Accepted 18 May 2004

\begin{abstract}
A detailed analysis of high-resolution spectra obtained in three different wavelength regions (at $430 \mathrm{~nm}, 526 \mathrm{~nm}$ and $569 \mathrm{~nm}$ ) of $G$-band bright points in the solar photosphere is presented. They show an average intensity contrast of $11 \%$ with respect to the "quiet" sun reference. The $\mathrm{CH}$ lines are weakened in the bright point interior. The atomic lines, too, e.g. the Fe I line at $569.15 \mathrm{~nm}$, weaken in the bright point interior. In contrast thereto, the absorption line of single ionized iron at $526.48 \mathrm{~nm}$ remains almost constant between bright point interior and the immediate surroundings. Line-of-sight velocities show a stronger downflow within bright points than in the close environment. A net downflow relative to the intergranular surroundings of around $80 \mathrm{~m} / \mathrm{s}$ is measured. Filling factors are calculated from a comparison with synthesized spectra for different flux tube models and are used to estimate the "true" velocity in bright points with respect to their immediate surroundings. We obtain up- and downflows in the order of one $\mathrm{km} \mathrm{s}^{-1}$, in agreement with the magneto-convective picture of the formation and dispersal of magnetic flux tubes. From the different behavior of the metallic lines and the $\mathrm{CH}$ lines we conclude that the line-weakening process that leads to the $G$-band bright points is mainly due to hot-wall radiation. This confirms that these bright points are indeed magnetic flux elements.
\end{abstract}

Key words. Sun: photosphere

\section{Introduction}

When the sun is imaged in the $G$ band (spectral range at $430 \mathrm{~nm}$ dominated by electronic transitions of the $\mathrm{CH}$ molecule) and at sufficiently high angular resolution the $G$-band bright points become visible (Muller \& Roudier 1984; Muller \& Mena 1987; Title \& Berger 1996; Berger 1997; Berger et al. 1998). G-band bright points are generally associated with magnetic flux tubes (cf. Berger \& Title 2001). Hence, $G$-band images provide a useful proxy diagnostic tool for photospheric magnetic flux tubes. The physical basis of this proxy magnetometry is not completely solved.

In the basic theoretical picture of an isolated flux tube in magneto-hydrostatic equilibrium the magnetic pressure inside the tube is balanced by a reduced density of its atmosphere relative to the surrounding non magnetic atmosphere. This leads to a reduced opacity. The iso-surfaces of optical depth are depressed at the location of the tube and hotter material of the exterior becomes exposed forming the "hot wall" (e.g. Spruit \& Zwaan 1981). The radiation from the side may affect the tube interior in two ways: heating of the tube interior through radiative influx and photo-dissociation of the $\mathrm{CH}$ molecule by UV-radiation from the hot wall. The latter was first conjectured by Rutten (1999) and further discussed by e.g. Rutten et al. (2001) and Sánchez Almeida et al. (2001). Rutten et al. (2001) conclude from LTE spectral synthesis based on a standard flux tube model that the evacuation of the flux tube leads to execss $\mathrm{CH}$ depletion within the tube. This results in a relatively more transparent flux tube, through which radiation from hot subsurface layers can escape. The $G$-bands contrast increases over the continuum due to an "enhanced hot wall effect". Further model calculations that relate $G$-band brightness and magnetic flux concentrations (all based on semi-empirical models) reproduce reasonably the observed bright point contrasts as well (Kiselman et al. 2001; Sánchez Almeida et al. 2001; Steiner et al. 2001). Steiner et al. (2001) note that $\mathrm{CH}$ depletion due to collisional dissociation in the deep photospheric layers alone would be a sufficient mechanism to explain the enhanced $G$-band contrast. Sánchez Almeida et al. (2001) conclude further that the $\mathrm{CH}$ concentration is not controlled by radiation. Ab-initio simulations of radiative magneto convection (Uitenbroek 2003; Schüssler et al. 2003) confirm this physical model of small scale magnetic flux concentrations as evacuated laterally heated structures. That the $\mathrm{CH}$ lines indeed 
Table 1. Top: specification of the spectral data. Bottom: specification of the image data. Abbreviations: FF - Flat Field, SNR - Signal-toNoise Ratio.

\begin{tabular}{lccc}
\hline \hline & $\lambda=430 \mathrm{~nm}$ & $\lambda=526 \mathrm{~nm}$ & $\lambda=569 \mathrm{~nm}$ \\
\hline Camera & 16 bit, $512 \times 512$ & 16 bit, $1024 \times 1024$ & 16 bit, $1024 \times 1024$ \\
Spectral order & 13 & 11 & 10 \\
Range [nm] & $430.23 \ldots 430.78$ & $525.93 \ldots 526.92$ & $568.34 \ldots 569.57$ \\
Continuum wavelength [nm] & 430.41 & 526.46 & 569.2 \\
Dispersion [pm/pixel] & 1.08 & 1.02 & 1.26 \\
Angular scale [arcsec/pixel] & 0.090 & 0.088 & 0.088 \\
Mean intensity continuum [counts] & $\sim 48750$ & $\sim 19600$ & $\sim 15200$ \\
Mean intensity line core [counts] & $\sim 10300$ & $\sim 5500$ & $\sim 9900$ \\
Detector noise [counts] & \pm 8 & \pm 18 & \pm 2 \\
FF rms-noise [\% of local intensity] & 0.86 & 0.97 & 0.56 \\
SNR continuum / line core & $75 / 52$ & $56 / 38$ & $72 / 63$ \\
Pore contrast in continuum [\%] & -27.1 & -26.8 & -24.1 \\
\hline & & Slit Jaw & "White light" \\
\hline Camera & & 12 bit, $1024 \times 1024$ & 10 bit, $1024 \times 1024$ \\
Bandpass filter & $1 \mathrm{~nm}$ at $430.5 \mathrm{~nm}$ & $10 \mathrm{~nm}$ at $480 \mathrm{~nm}$ \\
Angular scale [arcsec/pixel] & 0.041 & 0.039 \\
Mean intensity [counts] & & $\sim 15000$ & $\sim 7200$ \\
Detector noise [counts] & & \pm 11 & \pm 2 \\
FF rms-noise [\% of local intensity] & & 2.4 & 0.3 \\
SNR & & 31 & 65 \\
Pore contrast [\%] & & -31.5 \\
\hline
\end{tabular}

weaken in the spectrum of $G$-band brightenings was confirmed by Langhans et al. (2001) by recording the $G$-band spectrum from $430.24 \mathrm{~nm}$ to $430.78 \mathrm{~nm}$ of a few isolated $G$-band bright points. To measure the $\mathrm{CH}$ abundance variations from spectroscopic $G$-band data Langhans et al. (2002) defined the Bright Point Index (BPI) as the ratio:

$\mathrm{BPI}=\frac{I_{\mathrm{cont}}-I_{\mathrm{Fe}}}{\left\langle I_{\mathrm{cont}}-I_{\mathrm{Fe}}\right\rangle} \cdot \frac{\left\langle I_{\mathrm{cont}}-I_{\mathrm{CH}}\right\rangle}{I_{\mathrm{cont}}-I_{\mathrm{CH}}}-1$,

where $I_{\text {cont }}, I_{\mathrm{Fe}}$ and $I_{\mathrm{CH}}$ are the "continuum" intensity at $430.33 \mathrm{~nm}$, the line core intensity of Fe II $430.32 \mathrm{~nm}$ and of $\mathrm{CH} 430.32 \mathrm{~nm}$, respectively. The brackets denote spatial average over a reference region of quiet sun. The BPI allows to distinguish two classes of $G$-band bright points according to their spectroscopic signature: (1) structures that are bright in the $G$ band due to an increased local continuum intensity; (2) structures that are bright in the $G$ band due to a significant difference in $\mathrm{CH}$ - and Fe II-line depression. The latter are accompanied by downflows and do not have a conspicuous signature in the continuum.

With the observations presented in this paper we improved and extended our previous observations (cf. Langhans et al. 2001, 2002). Section 2 describes the observations, data characteristics and error sources. Section 3 deals with the method of analysis. Our results are presented and discussed in Sects. 4 and 5 .

\section{Observations}

\subsection{The data}

The data were acquired on October 6, 2001 at the Richard B. Dunn Solar Telescope (DST) of the National Solar Observatory
(NSO) at Sacramento Peak, Sunspot, New Mexico, USA. The data consist of high resolution spectra in three different wavelength ranges obtained with the horizontal spectrograph (HSG). Simultaneous slit jaw images were taken in $G$ band using a $0.92 \mathrm{~nm}$ bandpass filter centered at $430.55 \mathrm{~nm}$. Broadband images, in the following refered to as "white light" images, were also obtained using an interference filter with a bandpass of $10 \mathrm{~nm}$ centered at $480 \mathrm{~nm}$.

The HSG slit width was set to $40 \mu \mathrm{m}$ corresponding to 0.3 arcsec. Spectral maps of $40 \times 10.5 \operatorname{arcsec}^{2}$ (slit length 40 arcsec) were obtained by repeated spatial scanning of the spectrograph slit across the region of interest. The step size was 0.3 arcsec. The detailed observing parameters are given in Table 1. The NSO adaptive optics (AO) system (Rimmele et al. 1999; Rimmele 2000) was correcting the low order wave front aberrations. The system was tracking on a pore at heliographic position $\mathrm{N} 20.2^{\circ} \mathrm{W} 12.3^{\circ}$ corresponding to $\mu=0.95$. An integration time of $1.5 \mathrm{~s}$ was required to achieve a sufficient signal-to-noise ratio of the $G$-band spectra. The spectra at $526 \mathrm{~nm}$ and $569 \mathrm{~nm}$, the broad band and slit jaw images were taken with the same integration time as the $G$-band spectra. The combination of white light image and $G$-band slit-jaw images allowed to identify the bright points (cf. Berger et al. 1998).

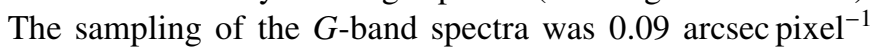
along the slit and $1.08 \mathrm{pm} \mathrm{pixel}^{-1}$ in wavelength.

Dark current subtraction and flat field corrections were done separately for each type of data. The spectra at $526 \mathrm{~nm}$ and $569 \mathrm{~nm}$ as well as the broadband continuum data and the slit jaw data were scaled and aligned with respect to the $G$-band spectra using images of a resolution target to determine image scale factors and image offsets. In Figs. 1 and 2 the common field-of-view (FOV) is displayed for the different data sets. 


\subsection{Data quality and error estimation}

Three scans, obtained during variable seeing conditions, were analyzed. In the spectro-heliograms in Fig. 2 individual scan steps are visible as columnar structures. Near the end of the scan, the AO system lost tracking and the image contrast decreased immediately. A number of bright points are visible at $y=11$ as a horizontal bright line in the spectral data; this conglomerate of bright points is better resolved in the slit jaw image in Fig. 1 where three individual $G$-band bright points are visible. This scan contains 15 consecutive exposures with sufficient image quality (marked by solid lines in Fig. 2).

The data quality decreases continuously with distance from the tracking point (Fig. 1) The dashed box in Fig. 2 e marks the region, where the image quality in the broadband channel is close to the resolution limit of the telescope. The common area of both marked frames is used for further analysis. For the spectral data, the sampling along and perpendicular to the slit is given by the pixel size of the detector $(0.2 \mathrm{arcsec})$ and the slit width $(0.3$ arcsec $)$, respectively.

The measurement error of the observed broadband images consists of detector and photon noise and a contribution due to imperfect flat field correction. The same errors contribute to the error budget of the spectral data (cf. Table 1). The contribution of spatially scattered light within the spectrograph was estimated to $8.7 \%$ by a comparison of the contrast of the pore in the white light image and in the spectra. We estimate the contribution of spectral scattering to $4 \%$ in the line core, by comparing a high-resolution atlas-spectrum Wallace et al. (1998), convolved with the instrument profile of the HSG, to the observed data. A detailed error discussion can be found in Langhans (2003).

\section{Data reduction and analysis}

The broadband images were used to measure the intensity variations along the slit. Since the slit jaw image that is taken simultaneously with the spectra is useless for this purpose, we select an image pair - slit jaw and broadband continuum - within an interval of $15 \mathrm{~s}$ with respect to the spectral data as broadband reference. Due to the lack of strict simultaneity to the spectral data, the broadband intensity along the slit is used for identification of $G$-band bright points only. The intensity contrast $C$ is defined as the intensity $I$ normalized to the average intensity of the reference area (as marked in Fig. 1) minus 1.

$C=\frac{I}{\langle I\rangle}-1$.

The line core intensities and positions (and hence the Doppler velocity) were derived by locating the minimum of a 2nd-order polynomial fit to the spectral lines. Equivalent widths are evaluated by profile integration. In the crowded $G$ band, the adjacent intensity maxima are used to define the local continua and profile extent. The BPI was then computed from the continuum and line core intensities, using Eq. (1). We use the upper third of the common FOV as a reference for the velocity measurements. Since we do not have a time series, solar oscillations may influence our results, thus a comparison of velocities is only valid within distances of a few arcsec.

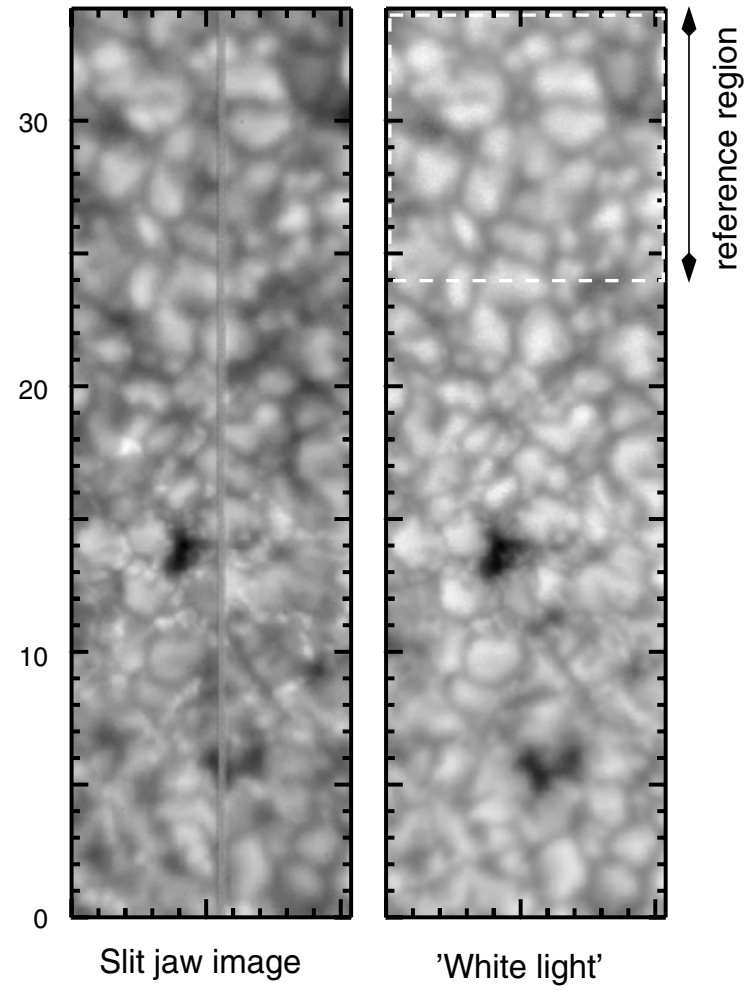

Fig. 1. Broadband data. One tick mark corresponds to 1 arcsec. Left: $G$-band image (broadband $1 \mathrm{~nm}$ at $430.5 \mathrm{~nm}$, slit jaw camera), ritght: white light image (broadband $10 \mathrm{~nm}$ at $480 \mathrm{~nm}$ ). Dashed box region of reference.

Bright point identification is often based on the difference method developed by Berger et al. (1998). However, the spectral data presented here may offer more and better ways to identify bright points. For example, the difference of continuum intensity at $430.41 \mathrm{~nm}$ and the integrated $G$-band intensity or, alternatively, the $\mathrm{CH}$-line core intensity can be used in order to enhance the contrast for easier identification of bright points. The BPI is used as an additional indicator to distinguish the brightenings of the two classes of bright points as defined by Langhans et al. (2002).

Physical properties of bright points are determined from our one-dimensional spectral data separately for the bright point interior and its surroundings. The interior of a bright point is defined as follows: data points with a BPI-value above two thirds of the maximum BPI define the size, $S$, of the bright point. The adjacent data points within a range of $\pm 0.5 S$ define the immediate surroundings. This definition is illustrated in Fig. 4 (dashed lines).

\section{Results}

A total of 46 structures were identified as bright points in the three analyzed scans. The time between scans is $150 \mathrm{~s}$ and $600 \mathrm{~s}$. Since the scans cover partly the same area, 7 structures appear twice in the sample. They are nevertheless counted as different structures.

Spatial averages over all bright points within the sample were calculated for the interior and the immediate 

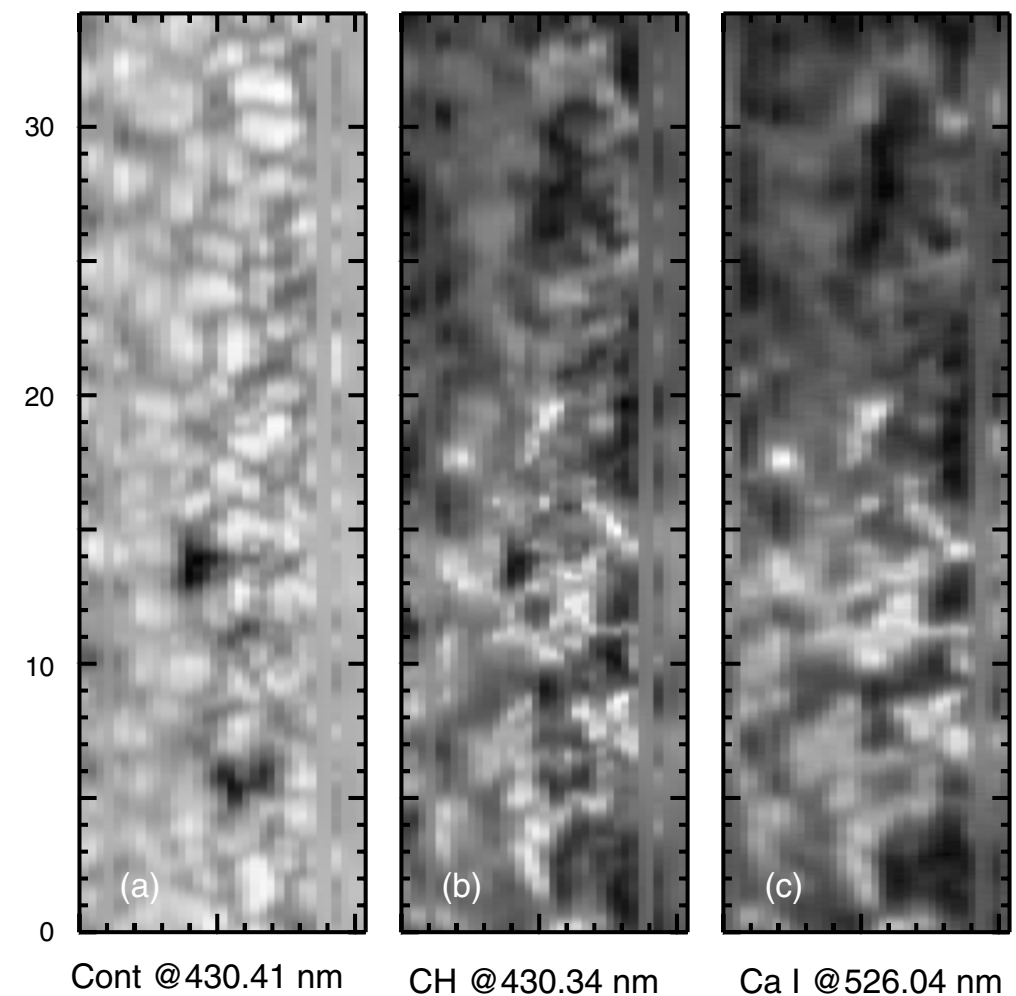

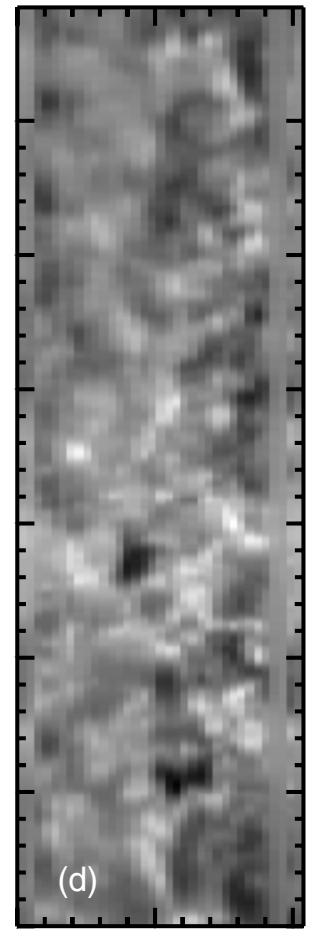

Fe I @ 569.15 nm

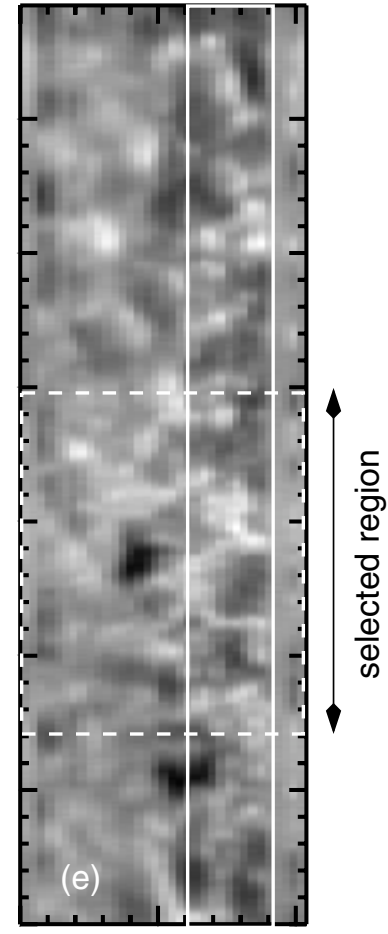

Fe II @526.48 nm

Fig. 2. Spectral data. Example for one scan. One major tickmark corresponds to 1 arcsec. a) Continuum intensity at $430.41 \mathrm{~nm}$, b)-e) Line core intensities of the analyzed absorption lines. Dashed box - region around tracking point of AO system with sufficient correction, solid box exposures of high quality used for analysis.

Table 2. Averaged properties of bright points, based on the spectral data (Int.: interior; Surr.: surroundings; Ref.: reference).

\begin{tabular}{lccc}
\hline \hline & Int. & Surr. & Ref. \\
\hline$G$-band contrast [\%] & 11.0 & 6.2 & 0 \\
Bright Point Index & 0.248 & 0.142 & 0 \\
Cont. contr. at 430.41 nm [\%] & -4.2 & -4.1 & 0 \\
Cont. contr. at 569.20 nm [\%] & 1.7 & 0.8 & 0 \\
Cont. contr. at 526.46 nm [\%] & -1.4 & -1.9 & 0 \\
Line depression CH & 0.46 & 0.51 & 0.60 \\
Line depression Fe I & 0.24 & 0.27 & 0.33 \\
Line depression Fe II & 0.39 & 0.40 & 0.43 \\
\hline
\end{tabular}

surroundings separately. The averaged bright point properties are listed in Table 2 . The bright point sizes range from 0.18 arcsec to 0.63 arsec. The brightest point in the sample has a contrast of $28.3 \%$ and the weakest bright point shows a contrast of $-2.3 \%$. While the bright points on average stand out from their surroundings, they show no significant enhancement in the mean continuum intensity contrast. In the average over all bright points within the sample the continuum intensity contrast of interior and surroundings is almost equal. This confirms that the brightness of the $G$-band bright points is mainly a line effect. In particular this effect is observed in the $\mathrm{CH}$ lines. The depth of the $\mathrm{CH}$ lines in the bright point interior is (on average) reduced by $23 \%$ compared to the quiet sun value. A similar value is obtained for the weak Fe I line at $569.15 \mathrm{~nm}$, while the line depression of the Fe II line is only slightly reduced.

\subsection{Individual cases}

The individual bright points discussed in this section are representative for the observed sample. The quantities illustrated in Fig. 3 have been explained in Sect. 3 .

The average value of all measured line widths and equivalent widths within the region of reference (as marked in Fig. 1) are used as reference. In Figs. 3a,b the slit jaw and white light images of the selected bright points are displayed. The FOV is $3.6 \times 4.2 \operatorname{arcsec}^{2}$; one tick mark corresponds to 0.25 arcsec. We note that these images were not recorded strictly simultaneous with the spectral quantities shown in the graphs (Figs. $3 \mathrm{~d}-\mathrm{k}$ ). In the slit jaw images of the examples 1, 3 and 6 the slit is visible as a uniform gray bar across the image. Black lines indicate the slit position for which the physical quantities were plotted in rows $\mathrm{c}$ to $\mathrm{k}$. The spatial range of the line plots is indicated by grey vertical lines.

The graphs in Fig. 3c show the intensity contrast of the white light (solid curve) and $G$-band images (dashed curve) along the slit. When intensity quantities based on spectral data are compared to quantities based on broadband intensities, the different spatial resolution should be taken into account. For clarity, the curves are displayed without error bars.

In the following we discuss properties of the selected individual bright point examples.

- Example 1 is a single isolated intergranular bright point that is well visible both in white light and the $G$-band image. In the $G$-band image (row a) it appears as two neighbouring very small brightenings indicating that there may be 

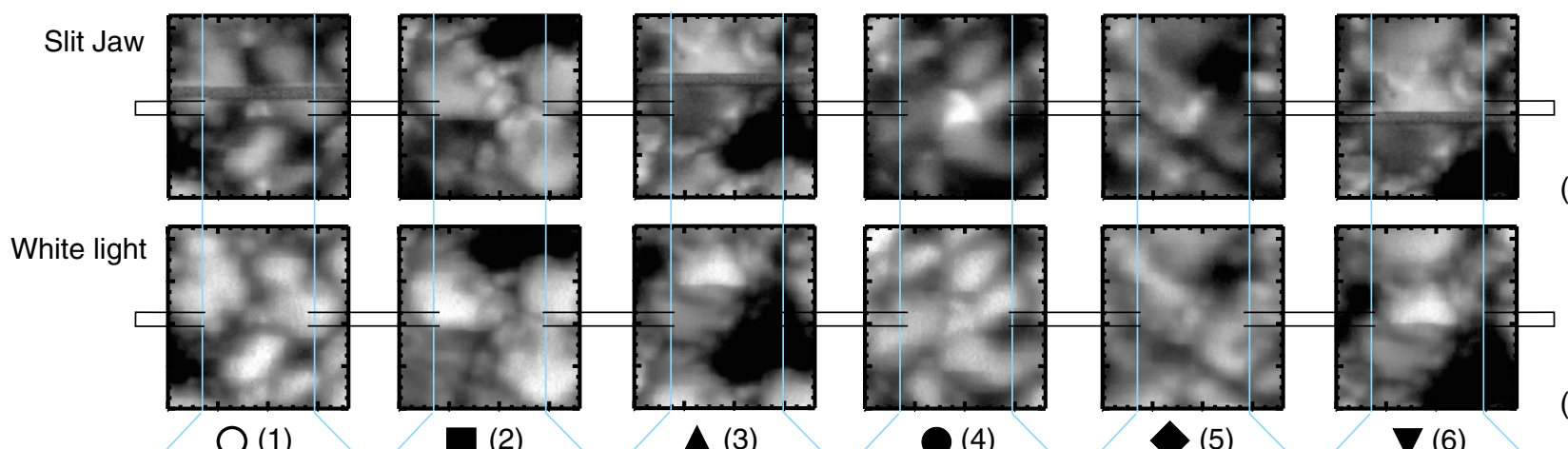

(a)
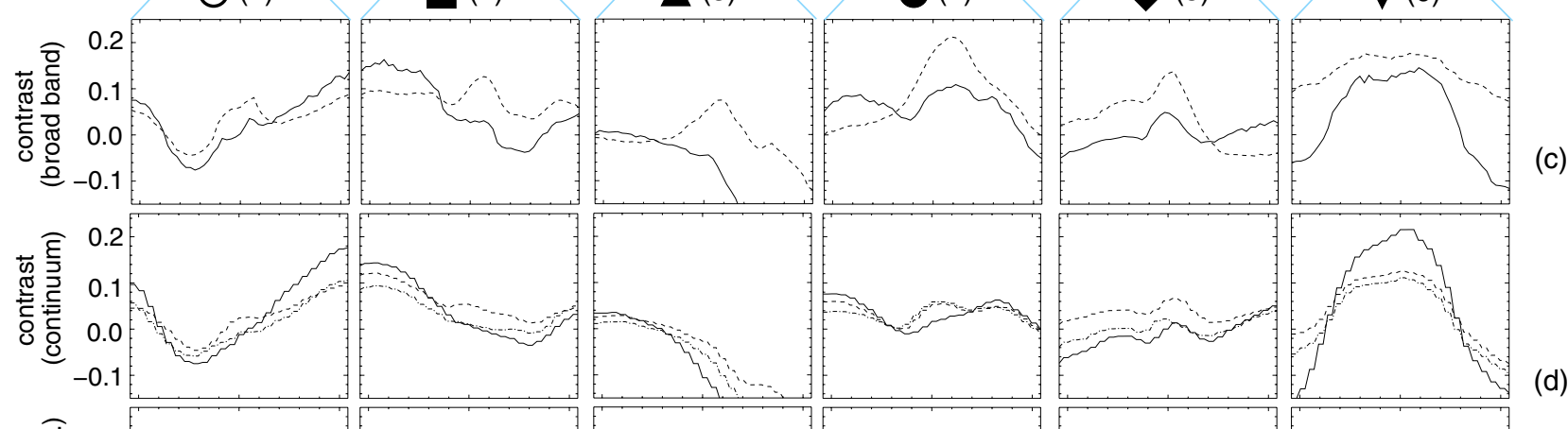

(c)
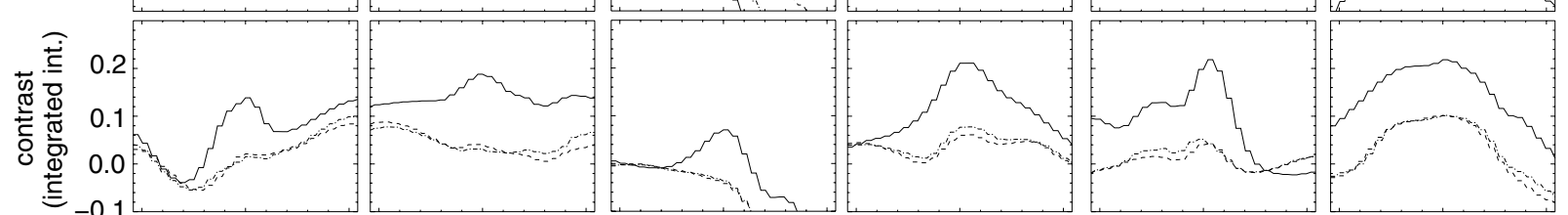

(d)
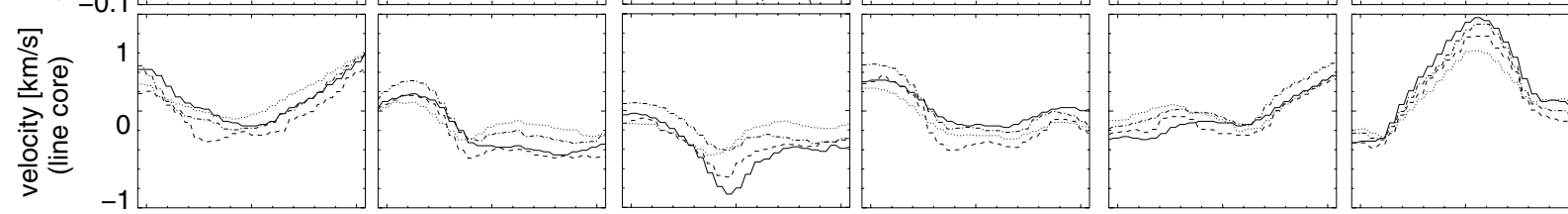

(e)
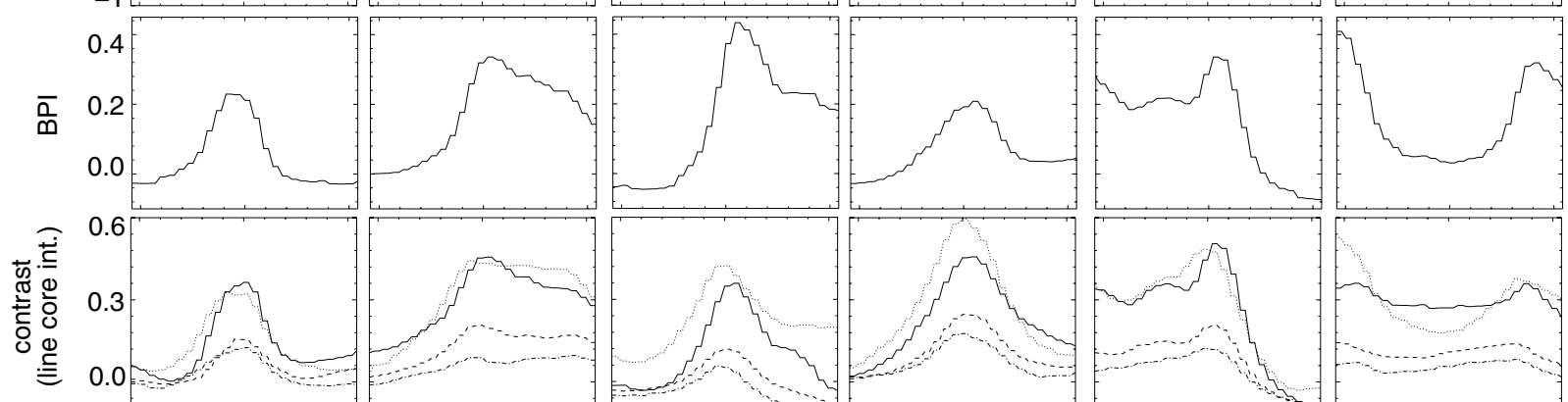

(f)
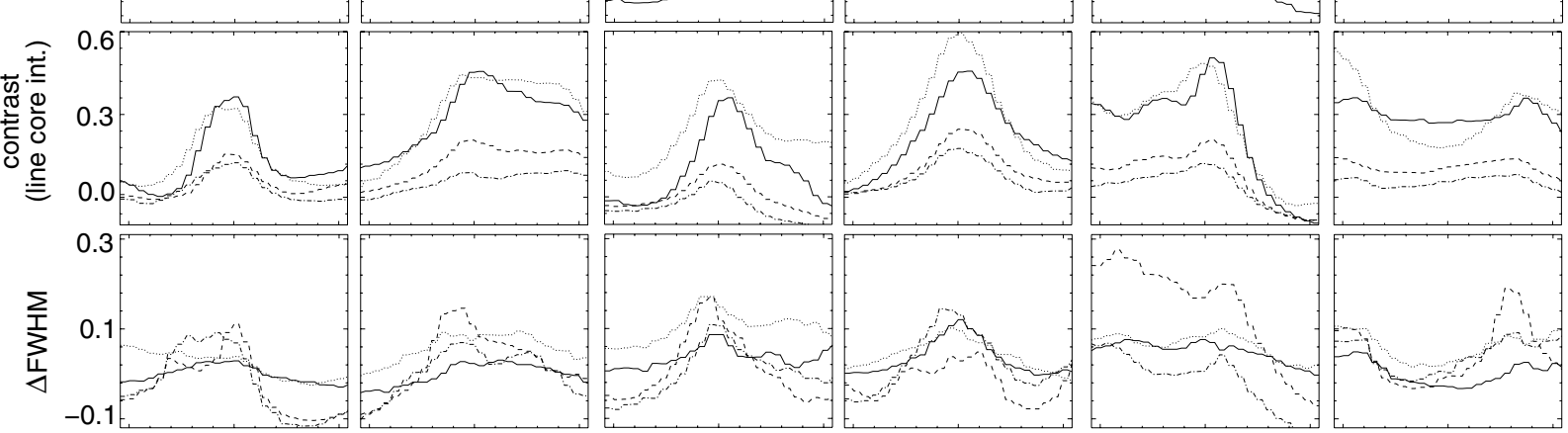

(g)
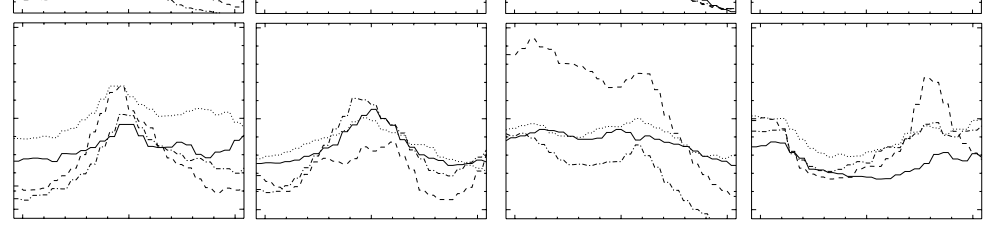

(h)
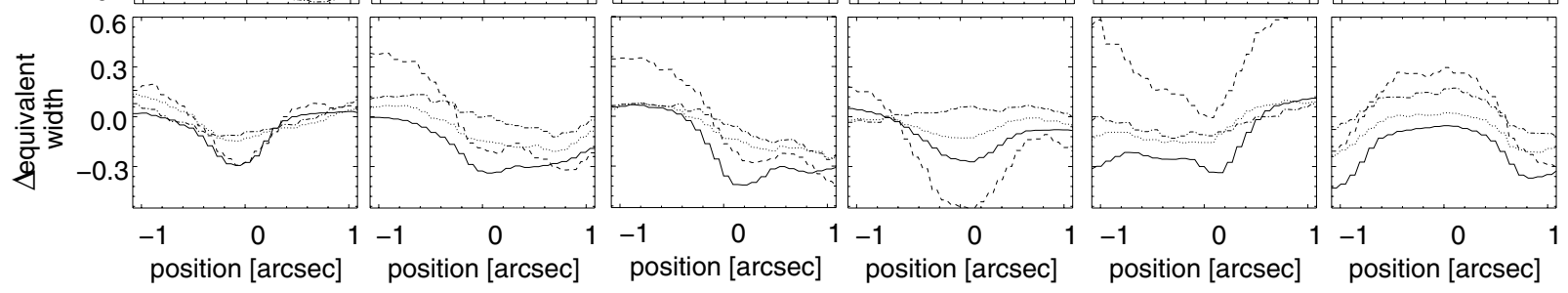

(b)

(c)

)

\section{(1)}

(g)

Fig. 3. Individual Case Studies. a), b) Broadband images of the bright point examples in $G$-band (slit jaw images) and "white light". c) Variation of $G$-band (dashed curve) and "white light" intensity contrast along the slit (solid curve), taken from the broadband images. d) Continuum contrast. e) Contrast of integrated intensity. f) Doppler velocity. g) Bright Point Index. h) Variation in line core intensity. i) Variation in FWHM. k) Variation in equivalent width. Curve coding for d)-k): solid - CH, dashed - Fe I, dashed-dotted - Fe II, dotted - Ca I. 

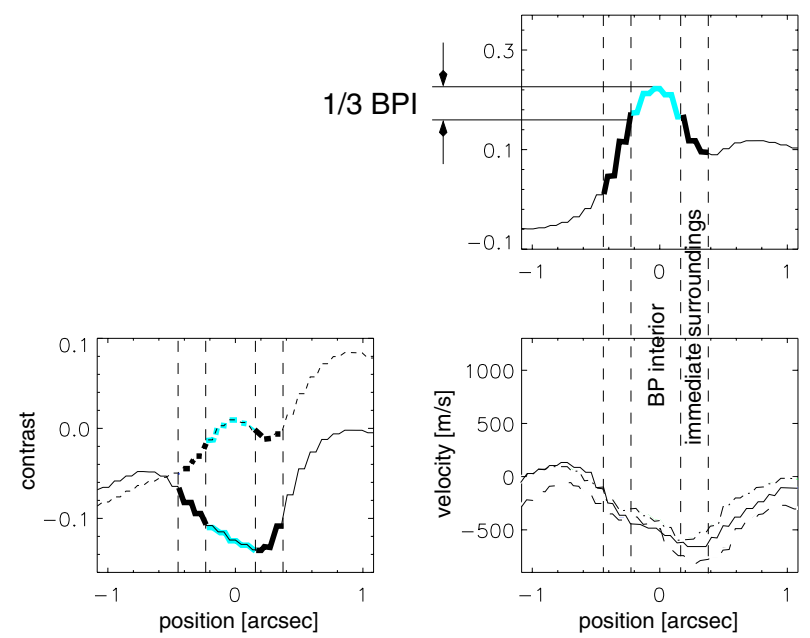

Fig. 4. Illustration of the velocity measurements. Vertical dashed lines indicate the borders of bright point interior and immediate surroundings, respectively. Top: BPI. Bottom left: intensity contrast, continuum intensity (solid), integrated $G$-band intensity (dashed). Bottom right: flow velocity.

unresolved substructure. In the broadband contrast graph (row c) this separation becomes blurred due to the averaging over the slit width. The variation in "white light" contrast (row c, solid curve) is small in comparison to the immediate surroundings and far below the contrast of the nearby granule. This is also visible in the continuum intensity contrast (row d). In the integrated $G$-band intensity (row e, solid curve) the bright point contrast rises above the surroundings. This demonstrates that the brightening is a line effect. The size, $S$, of this bright point is about 0.3 arcsec.

- Example 2 is a bright point located at the edge of a granule. It is visible in the white light image but much less conspicuous than in the $G$-band image. The maximum of the BPI coincides with the location of the bright point. The BPI decreases immediately toward the granular side of the bright point but slowly toward the intergranular side. This is possibly due to the nearby bright point visible in the slit jaw image. The line core intensity of the $\mathrm{CH}$ and the $\mathrm{Ca}$ I line show a similar behavior.

- Example 3 is an isolated bright point located at the edge of a pore. In this example the difference in contrast between $G$-band and continuum intensity is most pronounced. Both $G$-band and continuum contrast values decrease toward the pore. At the location of the bright point the $G$-band intensity contrast is highest, whereas the continuum intensity contrast decreases. In the variation of the broadband continuum contrast this structure is hardly visible. The Doppler velocity shows a strong downflow at the perimeter of the pore (cf. Sütterlin et al. 2001; Tritschler et al. 2002; Sankarasubramanian \& Rimmele 2003). This downflow coincides with the bright point position. The BPI of this bright point is one of the highest measured in the analyzed sample. Toward the pore the BPI decreases but remains enhanced at the pore location.
- Example 4 is the largest isolated intergranular bright "point" within the sample. This large bright point possibly an unresolved accumulation of bright points - differs from smaller bright points interm of the velocity measured within the bright point. The bright point is embedded in the downflow of an intergranular lane but itself shows an upflow relative to its immediate surroundings (cf. Fig. 5). This large bright point - possibly an unresolved accumulation of bright points - is embedded in the downflow of an intergranular lane but itself shows an upflow relative to its immediate surroundings (cf. Fig. 5).

- Example 5 represents a group of bright points with a more diffuse signature in the broadband images. These brightenings neither clearly belong to a granule nor to intergranular lanes but are identified as bright points with the selection method used here. The group of bright points shows similar spectral properties as the examples discussed before. This example shows also a significant signature in the contrast of the integrated intensity in the wavelength ranges at $526 \mathrm{~nm}$ and $569 \mathrm{~nm}$.

- Example 6 shows a large variation in the intensity contrast in the $G$ band compared to the "white light" but does not have an enhanced BPI. The bright object, obviously a granule, is accompanied by small structures with enhanced BPI that are visible only in the $G$-band images. The continuum intensity enhancement is limited to the granule (d). The $\mathrm{CH}$ - and CaI-line core intensity show the opposite slope: lower in the middle and enhanced toward the outside. Consequently the BPI is low for the granule and high for the small companions. The granule is related to a strong upflow while the nearby small structures have negligible velocities.

Those bright points with enhanced BPI have a reduced equivalent width of the $\mathrm{CH}$ and the $\mathrm{Fe}$ I lines, whereas the equivalent width of the Fe II line remains almost constant. The FWHM of the $\mathrm{CH}$, the $\mathrm{Fe}$ I and the Fe II lines increase at bright point locations. The increase is strongest for the Fe I line.

\subsection{Doppler velocities}

Langhans et al. (2002) have shown that the bright points with an enhanced BPI are embedded in downflow regions. The comparison of the averaged interior and surroundings velocities for all bright points within our sample confirms those observations and in addition shows that the downflow within the bright point interior is stronger than in the immediate surroundings by about $73 \mathrm{~m} \mathrm{~s}^{-1}$ on average.

The location of a bright point does neither necessarily coincide with the minimal continuum intensity nor with the strongest downflow in the intergranular lane. The example shown in Fig. 4 illustrates this. Due to insufficient resolution the velocity measurements in the interior and, espacially, in the immediate surroundings of this bright point are most likely contaminated by intergranular velocities on "both sides" of the bright point. A clean separation of bright point velocity and intergranular velocity is therefore not possible. In addition, the influence of projected horizontal motions cannot be ignored: at the disk position of our observations $(\mu=0.95)$ a horizontal flow has a line-of sight component of $31 \%$. 

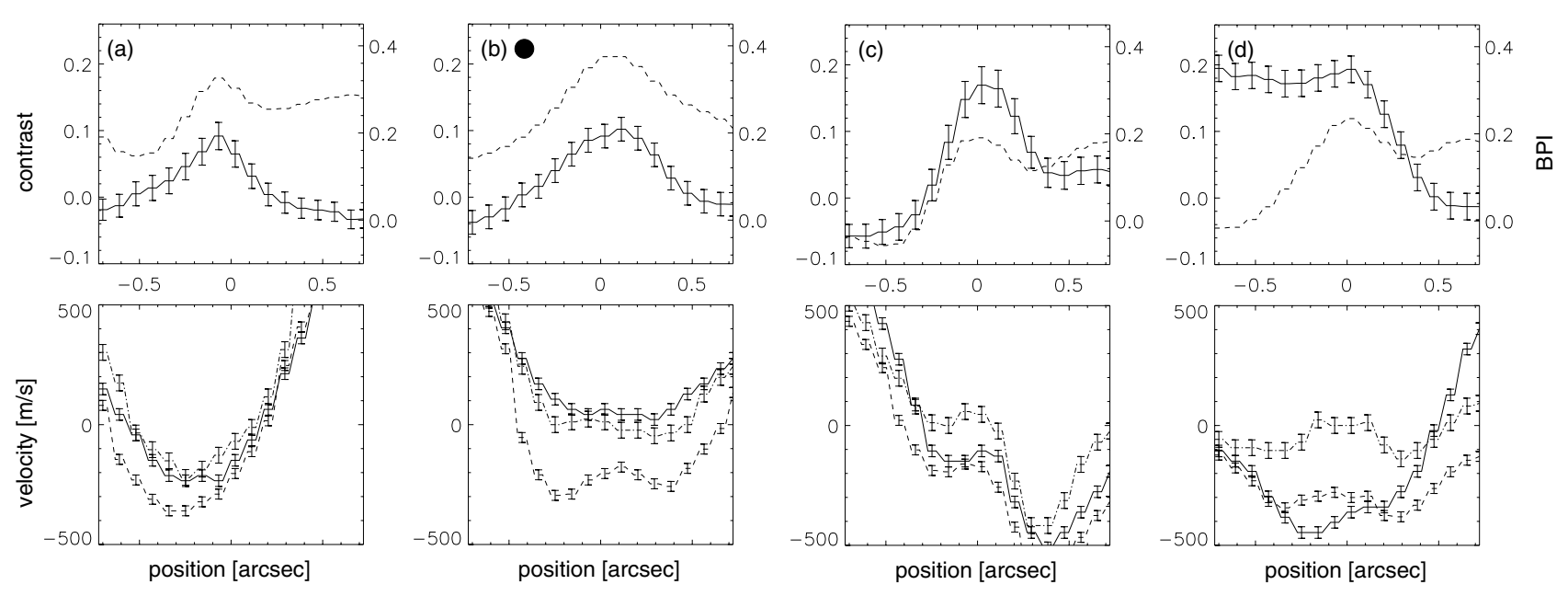

Fig. 5. Four examples of velocity measurements of bright points. Top: course of the BPI (solid curve, scale to the right) and the $G$-band intensity contrast (dashed curve, scale to the left). Bottom: velocity measurements (solid - CH, dashed - Fe I, dashed-dotted - Fe II). The plotted error bars correspond to the $1 \sigma$-level. b) Is again Example 4 of Sect. 4.1 .

Some bright point interiors show a clear velocity enhancement in comparison to their immediate surroundings. Examples are shown in Figs. 5b-d. Figure 5a shows a bright point without any velocity variation correlated to BPI enhancement. Instead the velocity variation is smooth and is typical for an intergranular lane. Figures $5 \mathrm{~b}-\mathrm{d}$ show significant variations of the lineof-sight velocity correlated to a BPI enhancement. While in Fig. $5 b$ the change is more clear for the measurement based on the Fe I line shift, in Fig. 5d the velocity based on the Fe II-line shift shows the most significant variation toward an upflow or at least a reduced downflow in the bright point interior.

\section{Discussion}

\subsection{Comparison to $2 D$-spectroscopic data}

The $\mathrm{CH}$ and Fe II line used for the two-dimensional observations of Langhans et al. (2002) are within the same wavelength range covered by the spectroscopic observations of this work. Consequently we were able to perfom the same analysis done by and a direct comparison with the results published by Langhans et al. (2002).

In Fig. 6 we compare the distribution of narrow-band continuum contrast, $\mathrm{CH}$-line core contrast and velocity vs. the BPI index for both the two-dimensional observations and the spectroscopic (one-dimensional) data. The data points plotted in these scatter diagrams were selected according to the criterion: intensity contrast $C_{G \text { band }}=\frac{I}{\langle I\rangle}-1 \geq 0$. The grey points represent the two-dimensional and the crosses the onedimensional observations. In both cases the the point clouds show very similar distributions. The error ellipsoids in the correlation diagrams represent the rms-values of the total error for the the one-dimensional data only. The symbols refer to the examples described in Sect. 4.1. The one-dimensional spectroscopic observations confirm the results of the two-dimensional spectral data, which had slightly lower spatial resolution but better statistics. Data points with a BPI around 0 correspond mainly to granules and show no line core contrast, a slightly enhanced continuum contrast and are related to upflows with respect to the reference. With increasing BPI the line core contrast increases while the continuum contrast and the flow velocity decrease.

In comparison to the two-dimensional data the onedimensional data produces slightly lower values of the line core contrast. This might be due to better spectral and spatial resolution achieved with the one-dimensional data, which results in a larger filling factor. A larger filling factor in turn causes an increase in the $\mathrm{CH}$-line core intensity within a bright point.

\subsection{Comparison to synthetic spectra}

In this section the results of our one-dimensional spectroscopic observations are discussed and compared to synthetic spectra, which were calculated using different model atmospheres: FAL F - bright network/faint plage, FAL P - bright plage (both Fontenla et al. 1999) and net - network elements (Solanki \& Brigljević 1992). In order to compare synthetic spectra to observed ones the synthetic line profiles are convolved with the instrument profile of the HSG. Starting with the synthetic spectra computed from the different atmospheric models spectral quantities were calculated in the same way as described in Sect. 3 for the observed spectra. The reference spectrum for the synthetic spectra is based on the model FAL C (average supergranule cell interior, Fontenla et al. 1999).

The simulated and observed spectral quantities are plotted in the diagrams of Fig. 7. The bars indicate the range of values observed for bright point interiors. We conclude that the bright network model (FAL F) does not represent the observations of bright points. The bright network model predicts a rather low $G$-band contrast and a negative BPI, whereas observed bright points are characterized by a positive BPI. We note that due to spatial insufficient resolution the real $G$-band contrast is likely significantly higher than what we observe in our spectra, i.e., the discrepancy between bright network model and observations would be even more severe. 

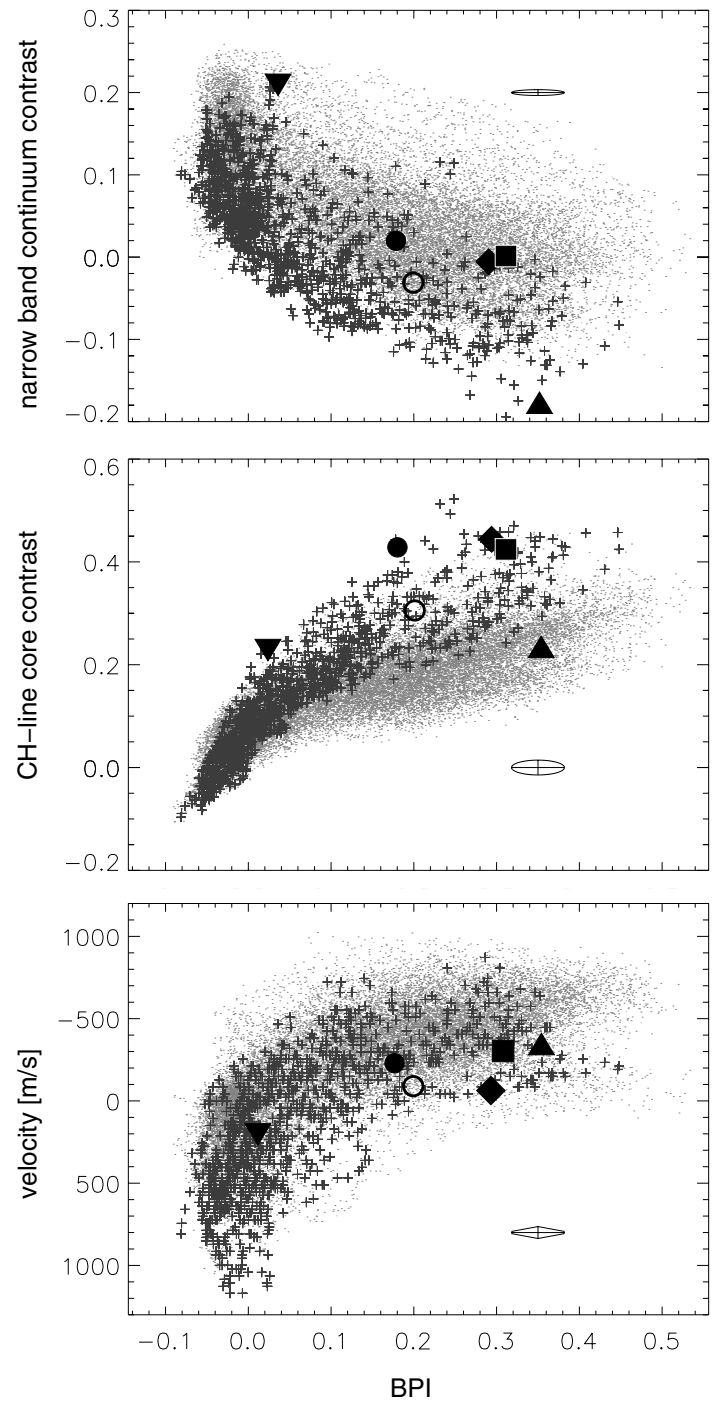

Fig. 6. Correlation diagrams. Criterion of data point selection is a $G$-band contrast $C_{G \text { band }} \geq 0$. The grey point clouds refer to the distribution of the two-dimensional data, black crosses to the onedimensional data. Different symbols mark the examples, described in Sect.4.1. The error ellipsoids indicate the $2 \sigma$-level for each data point. Top: continuum contrast vs. BPI (continuum at $430.41 \mathrm{~nm}$ ), Middle: $\mathrm{CH}$-line core contrast vs. sBPI. Bottom: flow velocity vs. BPI.

Both the $G$-band intensity contrast $C_{G}$ band as well as the BPI have the value 0 for the reference spectrum of the quiet sun and the FAL C-spectrum, respectively. Consequently both quantities can be used to calculate filling factors $\alpha^{\text {model }}$ based on the two remaining atmospheric models:

$\alpha_{\mathrm{C}}^{\mathrm{FALP}}=\frac{C_{G \text { band, synth. }}^{\mathrm{FALP}}}{C_{G \text { band, obs. }}}$ and $\alpha_{\mathrm{C}}^{\mathrm{net}}=\frac{C_{G \text { band, synth. }}^{\text {net }}}{C_{G \text { band, obs. }}}$

respectively

$\alpha_{\mathrm{BPI}}^{\mathrm{FALP}}=\frac{B P I_{\text {synth. }}^{\mathrm{FALP}}}{B P I_{\mathrm{obs}}}$ and $\alpha_{\mathrm{BPI}}^{\mathrm{net}}=\frac{B P I_{\text {synth. }}^{\mathrm{net}}}{B P I_{\mathrm{obs}}}$.

The resulting filling factors are listed in Table 3.

The FAL P-model for a bright plage, which assumes unresolved magnetic elements, produces larger values for the BPI
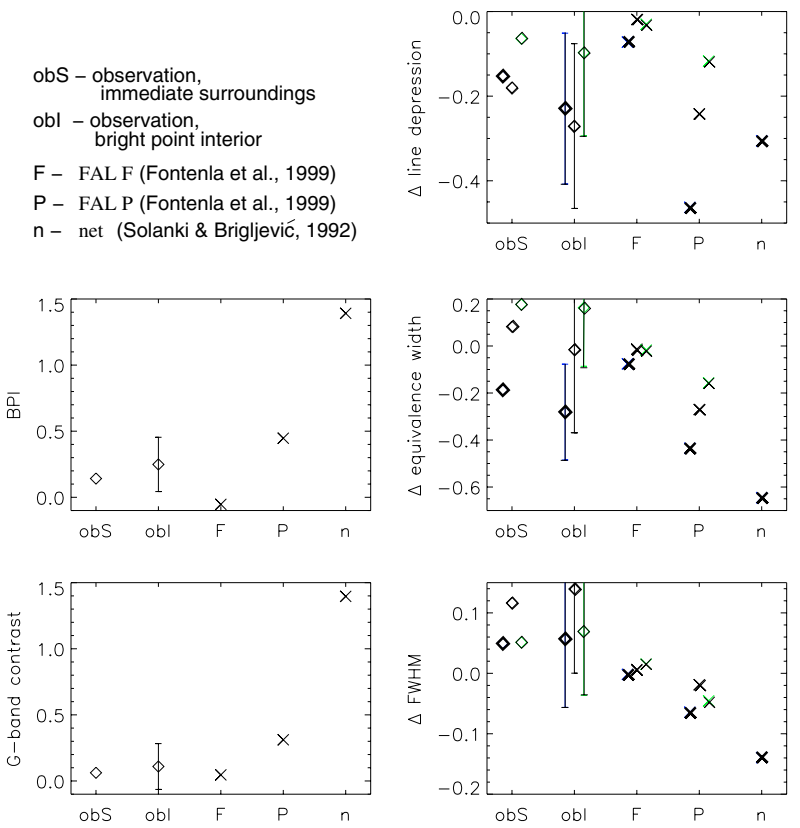

Fig. 7. Comparison of selected properties derived from synthetic spectra to observables. The bars indicate the range of values, observed for the bright point interior. Data points shifted to the left (thick symbols) refer to the $\mathrm{CH}$ line, data points shifted to the right (thin symbols) to Fe II and the centered symbols refer to Fe I.

and the $G$-band intensity contrast than the observations do. In addition, the line depression and equivalent width show a larger reduction with respect to the reference model than what is observed.

Regarding the range of observed values, especially for the brighter bright points (large $G$-band contrast and high BPI), the observables match well with the FALP-model predictions. We note again that due to insufficient resolution $G$-band contrast and BPI are likely underestimated in the observations.

The fairly good agreement between model and observations is also reflected in the computed filling factors $\alpha_{\mathrm{C}}^{\mathrm{FALP}}$ and $\alpha_{\mathrm{BPI}}^{\mathrm{FALP}}$. The filling factors are close to unity for the maximum observables and 0.35 for the averaged observed $G$-band contrast and 0.55 for the averaged BPI, respectively.

The net-model represents an atmosphere of an isolated magnetic element with a hot atmosphere. This results in a strongly enhanced continuum intensity (cf. Steiner et al. 2001). The extraordinarily high BPI and $G$-band intensity contrast produced by this model is mainly caused by the large increase of the continuum intensity (cf. Table 3 ). The change in the $\mathrm{CH}$-line depression is of the same order of magnitude as for the FAL P-model. The highest continuum intensity at $430.31 \mathrm{~nm}$ for an observed bright point reaches $7.9 \%$ of the continuum intensity predicted by the net-model.

In order to estimate an upper boundary for the actual observed continuum intensity we scale the observed contrast $(7.9 \%)$ with the lowest value for the computed filling factors $\left(\alpha_{\mathrm{C}}^{\text {net }}=0.08\right)$. This results in an upper limit for the actual intensity contrast of 0.76 , which is still below the continuum intensity predicted by the the net-model. This might be an indication that the net-model is actually too hot. 
Table 3. Top: continuum contrasts, $C_{\text {cont }}$, derived from synthetic spectra, with respect to the FAL C-model atmosphere. The listed values from observation refer to the average (maximum) over all observed bright point interiors with respect to the spectrum of the reference region. Bottom: filling factors derived from Eqs. (3) and (4).

\begin{tabular}{lcrrr}
\hline \hline & Observation & FAL F & FAL P & Net \\
\hline$C_{\text {cont }}(430.31 \mathrm{~nm})$ & $-0.04(0.14)$ & 0.00 & 0.09 & 0.77 \\
$C_{\text {cont }}(569.20 \mathrm{~nm})$ & $0.02(0.13)$ & 0.00 & 0.18 & - \\
$C_{\text {cont }}(526.46 \mathrm{~nm})$ & $-0.01(0.13)$ & 0.03 & 0.25 & - \\
\hline
\end{tabular}

\begin{tabular}{lcc}
\hline \hline Filling factors based on... & FAL P & Net \\
\hline .. G-band intensity contrast & $0.35(0.91)$ & $0.08(0.20)$ \\
.. Bright Point Index & $0.55(1.01)$ & $0.18(0.32)$ \\
\hline
\end{tabular}

\subsection{Upflows in magnetic elements?}

Previous velocity measurements of flux tubes are based on spectro-polarimetric data of unresolved magnetic elements (cf. Martinez Pillet et al. 1997; Sigwarth et al. 1999; Bellot Rubio et al. 2001). We present measurements that measure the flow velocities along the line-of-sight of bright points observed at high spatial resolution. Our analysis shows that bright points with different velocity signatures exist. The majority of bright points including their close environment show the velocity of a regular intergranular lane. All bright points with enhanced BPI are embedded in downflows in accordance with the result of Langhans et al. (2002). This includes also bright points located "on granules", such as example 2. The close environment of this particular bright point shows also a decreased line-of-sight velocity in comparison to the granule.

Taking the bright points as proxies for magnetic elements our measurements are in agreement with previous observations of red-shifted zero-crossing wavelengths of Stokes $V$ profiles in network and plage regions (e.g. Martinez Pillet et al. 1997; Sigwarth et al. 1999). A net downflow of $0.5 \mathrm{~km} \mathrm{~s}^{-1}$ was measured from the average of all observed Stokes $V$ profiles (cf. Sigwarth et al. 1999). For the smallest observed magnetic features the same authors observe "velocities of up to $5 \mathrm{~km} \mathrm{~s}^{-1}$ in both up- and downflows whereas for larger elements or clusters of several flux tubes the velocities become smaller and more uniform." Our sample of 46 bright points qualitatively gives the following results: both net downflows and upflows occur in individual bright points. However, the velocities we measured are much smaller than in the velocity measurements of Martinez Pillet et al. (1997) and Sigwarth et al. (1999), which refer only to the flow velocity of the magnetized part of the atmosphere.

Assuming that the observed bright points are the "white light" signature of magnetic elements and that the calculated filling factors $\alpha_{\mathrm{BPI}}^{\text {net }}$ and $\alpha_{\mathrm{C}}^{\text {net }}$ are correct the "real" velocity difference of interior and immediate surroundings can be estimated. This estimation is done in the following for two examples: (a) and (d) of Fig. 5. Example (a) coincides with a downflow. The maximum BPI amounts to 0.193 and the maximum $G$-band contrast to 0.179 . This results in filling factors $\alpha_{\mathrm{BPI}}^{\text {net }}=0.14$ and $\alpha_{\mathrm{C}}^{\text {net }}=0.13$, respectively. Taking these filling factors to scale the measured velocity difference $\Delta v_{\text {bis }}^{\mathrm{CH}}=$ $-100 \mathrm{~ms}^{-1}$ results in an actual velocity difference of $-700 \mathrm{~ms}^{-1}$ and $-750 \mathrm{~ms}^{-1}$, respectively. For example (d) the same estimation results in an upflow velocity of $1150 \mathrm{~ms}^{-1}\left(\alpha_{\mathrm{BPI}}^{\text {net }}=\alpha_{\mathrm{C}}^{\text {net }}\right)$ with respect to the immediate surroundings. (Calculation based on $\Delta v_{\text {bis }}^{\mathrm{CH}}=180 \mathrm{~m} \mathrm{~s}^{-1}$, the maximum BPI of 0.209 and a maximum $G$-band contrast of 0.211.)

A net downflow within magnetic elements is generally associated with the convective collapse of a magnetic element and according to models occurs over time periods of a few minutes during a formation of a $\mathrm{kG}$ strength flux tube. The upflow in magnetic elements might be explained by different processes that can be distinguished by their temporal evolution. Grossmann-Doerth et al. (1998) suggest that down-flowing gas might rebound in deeper layers and result in upward propagating shocks. Spruit \& Zweibel (1979) conclude from convective stability analysis that flux tubes with field strengths below a critical value $(\sim 0.13 \mathrm{~T})$ are unstable. The instability may result in either a downflow or an upflow. A downflow stabilizes the tube by intensifying the field strength correspondingly the super-adiabatic effect. An upflow reverses the process and causes flux tube dispersal. Flux tubes at this state of evolution might also be candidates for the observed bright points correlated with upflows.

Rimmele (2004) presents results of flow field measurements of magnetic elements based on diffraction limited filtergram and image data. He finds strong narrow downflows at the edges of many small magnetic flux concentrations, while the plasma within the flux concentration is in rest at layers in the photosphere represented by Fe I $630.2 \mathrm{~nm}$ and $557.6 \mathrm{~nm}$ dopplergrams. Although we measure on average an increased downflow in the bright point interior in comparison to its immediate surroundings, the observations of Rimmele (2004) are in correspondence with a number of bright points within the observed sample (cf. velocity diagrams of bright points 2, 4 and 5 in Fig. 3). Rimmele (2004) points out that his sample of flux elements likely consists mostly of stable flux concentrations located in the vicinity of a small sunspot. Dynamic processes such as convective collapse or upward propagating shocks may not be present in this sample. Telescopes of larger aperture are needed to provide more quantitative measurements of the velocity flow field of small scale magnetic flux concentrations with sufficient time resolution to capture the dynamics.

\section{Conclusions}

The brightening of $G$-band bright points, when properly distinguished from granulation brightenings, is mainly caused by a line effect. The line weakening within such a bright point is present in all observed neutral atomic lines but is pronounced for the absorption lines of the $\mathrm{CH}$ molecule due to the peculiarities of that molecule: (1) the low dissociation energy makes the $\mathrm{CH}$ molecule sensitive to many mechanisms that destroy the carbon-hydrogen compound. (2) The abundance of $\mathrm{CH}$ decreases in favour of $\mathrm{CO}$ with increasing height in the photosphere (Mount 1975). (3) Apart from this dependence 
between different compounds of carbon in the photosphere $(\mathrm{CO}, \mathrm{CN}$ and $\mathrm{CH}$ ), the concentration of $\mathrm{CH}$ (and other diatomic molecules as well) depends quadratically on the number density and is particularly sensitive to a reduction of the latter. In LTE the concentration of an atom that is appreciably ionized, such as the $\mathrm{Fe}$ I in the solar photosphere depends quadratically on density. "In the solar photosphere, however, ionization is already largely controlled by radiation, in which case the dependence is closer to linear" (Uitenbroek 2003).

This explains the extraordinarily strong weakening of the $\mathrm{CH}$ lines in magnetic flux tubes. The high line density of both $\mathrm{CH}$ lines of the $A^{2} \Delta-X^{2} \Pi$ transition and the variety of neutral atomic lines enhances the line weakening effect seen in the $G$ band. Therefore the $G$-band intensity is an excellent tracer of small scale magnetic field at the highest available spatial resolution.

The BPI is a reliable tool to quantify the spectroscopic signature of $G$-band bright points and allows for a classification of $G$-band brightenings. Since the BPI is based on relative intensities, it is virtually independent on the photometric properties of the instrument used.

A comparison of the observed spectra with synthesized spectra produced with the net- and the FAL P-model indicates that the temperature effect in the models is overestimated in comparison to the line effect. In contrast to the synthesized spectra the observed spectra do not show a strongly enhanced continuum intensity at the bright points' locations. Most spectroscopic properties (e.g. BPI, $G$-band contrast and equivalent width) derived from the bright plage model (FAL P), or the netmodel are within the range of the observed values (cf. Fig. 7). The modeled continuum intensities significantly exceed the observed values - especially for the net-model (cf. Table 3). These results indicate that the model atmospheres used are too hot. In part the observed continuum contrast might be caused by the fact that the small bright features are smeared out by the low intensity of the surrounding intergranular lanes due to the insufficient resolution. But the visibility of most bright points in the continuum and the high contrast observed in the integrated $G$-band intensity weaken this argument.

An additional hint that the direct temperature effect might be less important than others is given by the behavior of the ionized iron line at $526.48 \mathrm{~nm}$ and the $\mathrm{CH}$ line at $430.34 \mathrm{~nm}$ within the bright points. Based on the assumption that both lines form at similar height - justified by model calculations using the radiation transfer code provided by Uitenbroek (1998) - the brightness temperature obtained from the measured absolute intensity in the Fe II-line core can be identified with the temperature at $\tau=\mu=1$. The unchanged Fe II-line core intensity within a $G$-band brightening implies that the enhanced $\mathrm{CH}$-line intensity most likely is not due to a direct temperature effect - be it by means of the line source function or by enhanced dissociation of $\mathrm{CH}$. The $\mathrm{CH}$ dissociation is therefore most likely due to a process such as hot wall radiation. The temperature gradient of the applied standard fluxtube models imply a considerable energy dissipation at very low heights of the fluxtube atmosphere that is hard to explain. Sideways irradiation avoids this problem but will require NLTE. This idea is discussed in more detail by Rutten (2002).
This explanation implies that $G$-band bright points are indeed magnetic flux tubes. Spectropolarimetric measurements of the small-scale magnetic field in and around bright points, with sufficient spatial and temporal resolution are needed to confirm our conclusions. In addition, more extensive simulations and modeling efforts will be needed in order to be able to interpret the observations.

Acknowledgements. J. Bruls provided useful hints and comments. Oskar Steiner generously offered his synthesized $G$-band data of the net-model atmosphere. Han Uitenbroek provided his radiation transfer code. K. Langhans' research was funded by the Deutsche Forschungsgemeinschaft (grant No. SCHM 1168/3). Now he is supported by the European Community's Human Potential Programme through the European Solar Magnetism Network (contract HPRNCT-2002-00313). The DST data were obtained by K.L. as a visiting astronomer at the National Solar Observatory, operated by the Association of Universities for Research in Astronomy, Inc. (AURA), under cooperative agreement with the National Science Foundation.

\section{References}

Bellot Rubio, L. R., Rodríguez Hidalgo, I., Collados, M., Khomenko, E., \& Ruiz Cobo, B. 2001, ApJ, 560, 1010

Berger, T. E., Löfdahl, M. G., Shine, R. S., \& Title, A. M. 1998, ApJ, 495, 973

Berger, T. E. 1997, Ph.D. Thesis, Stanford University

Berger, T. E., \& Title, A. M. 2001, ApJ, 553, 449

Fontenla, J., White, O. R., Fox, P. A., Avrett, E. H., \& Kurucz, R. L. 1999, ApJ, 518, 480

Grossmann-Doerth, U., Schüssler, M., \& Steiner, O. 1998, A\&A, 337, 928

Kiselman, D., Rutten, R. J., \& Plez, B. 2001, in IAU Symp., 287

Langhans, K. 2003, Ph.D. Thesis, Universität Freiburg

Langhans, K., Schmidt, W., Rimmele, T., \& Sigwarth, M. 2001, in Advanced Solar Polarimetry - Theory, Observation, and Instrumentation, ASP Conf. Ser., 236, 439

Langhans, K., Schmidt, W., \& Tritschler, A. 2002, A\&A, 394, 1069

Martinez Pillet, V., Lites, B. W., \& Skumanich, A. 1997, ApJ, 474, 810

Mount, G. H. 1975, Ph.D. Thesis, University of Colorado

Muller, R., \& Mena, B. 1987, Sol. Phys., 112, 295

Muller, R., \& Roudier, T. 1984, Sol. Phys., 94, 33

Rimmele, T. 2004, ApJ, in press

Rimmele, T., Dunn, R., Richards, K., \& Radick, R. 1999, in High Resolution Solar Physics: Theory, Observations, and Techniques, ASP Conf. Ser., 183, 222

Rimmele, T. R. 2000, in Adaptive Optical Systems Technology, ed. Peter L. Wizinowich, Proc. SPIE, 4007, 218,

Rutten, R. J. 1999, in Third Advances in Solar Physics Euroconference: Magnetic Fields and Oscillations, ASP Conf. Ser., 184,181

Rutten, R. J. 2002, J. Astron. Data, 8, 8

Rutten, R. J., Kiselman, D., Rouppe van der Voort, L. H. M., \& Plez, B. 2001, in Advanced Solar Polarimetry - Theory, Observation, and Instrumentation, ASP Conf. Ser., 236, 445

Sánchez Almeida, J., Asensio Ramos, A., Trujillo Bueno, J., \& Cernicharo, J. 2001, ApJ, 555, 978

Sütterlin, P., Rutten, R. J., \& Skomorovsky, V. I. 2001, A\&A, 378, 251

Sankarasubramanian, K., \& Rimmele, T. 2003, ApJ, 598, 689 
Schüssler, M., Shelyag, S., Berdyugina, S., Vögler, A., \& Solanki, S. K. 2003, ApJ, 597, L173

Sigwarth, M., Balasubramaniam, K. S., Knölker, M., \& Schmidt, W. 1999, A\&A, 349, 941

Solanki, S. K., \& Brigljević, V. 1992, A\&A, 262, L29

Spruit, H. C., \& Zwaan, C. 1981, Sol. Phys., 70, 207

Spruit, H. C., \& Zweibel, E. G. 1979, Sol. Phys., 62, 15

Steiner, O., Hauschildt, P. H., \& Bruls, J. 2001, A\&A, 372, L13

Title, A. M., \& Berger, T. E. 1996, ApJ, 463, 797
Tritschler, A., Schmidt, W., \& Rimmele, T. 2002, in Solar Variability: From Core to Outer Frontiers, ESA SP-506, 477

Uitenbroek, H. 1998, ApJ, 498, 427

Uitenbroek, H. 2003, in Current Theoretical Models and Future High Resolution Solar Observations: Preparing for ATST, ASP Conf. Ser., 286, 403

Wallace, L., Hinkle, K., \& Livingston, W. 1998, An atlas of the spectrum of the solar photosphere from 13500 to $28000 \mathrm{~cm}^{-1}$ (3570 to $7405 \AA$ ) (Tucson, AZ: National Optical Astronomy Observatories) 